\title{
Hospital arrival time after onset of stroke
}

\author{
R Kay, J Woo, W S Poon
}

\begin{abstract}
To estimate the proportion of patients with stroke likely to be eligible for a trial of anticoagulant treatment for acute ischaemic stroke, the interval between onset of symptoms and arrival at hospital was analysed prospectively. Of 773 patients with stroke admitted in one year, $63 \%$ arrived at hospital within 12 hours, $76 \%$ within 24 hours, and $85 \%$ within 48 hours of ictus. The arrival time varied significantly with stroke subtype. Patients with intracerebral haemorrhage tended to arrive earlier than those with cerebral infarct, who arrived sooner than those with lacunar infarct. The results suggest that about half of all patients with ischaemic stroke in Hong Kong would present within 12 hours of ictus, in time for inclusion in a therapeutic trial.
\end{abstract}

(F Neurol Neurosurg Psychiatry 1992;55:973-974)

The opportunity to administer treatment within hours of ictus is crucial to various stroke therapy trials, which generally require the delay to be less than 48 hours. ${ }^{1-4}$ To estimate the proportion of patients likely to be eligible for inclusion in a trial of anticoagulant treatment for acute ischaemic stroke, an analysis of the interval between onset of symptoms and arrival at hospital was performed, on the basis of a series of patients with stroke, collected consecutively.

\section{Method}

The Prince of Wales Hospital, Shatin is the only acute general hospital serving a population of 931500 in the eastern New Territories of Hong Kong. The hospital is publicly funded and there are no private hospitals in the area. The hospital admits all cases of suspected stroke to the medical wards. In 1989, a survey was conducted of all patients presenting with stroke symptoms of less than seven days' duration and the Shatin Stroke Registry established.' Patients were examined within 24 hours of admission by a neurologist, who was responsible for verifying the diagnosis of stroke and determining its subtype according to the clinical and radiological criteria used by the Oxfordshire Community Stroke Project. ${ }^{67} \mathrm{CT}$ of the brain was performed on all cases unless the patient died before it could be arranged.
The time of onset of symptoms was obtained from the patient or any available witness; if the patient woke up with symptoms then the time of awakening was taken as the time of onset. Hospital arrival time, routinely noted, was the time that the patient arrived at the admissions office or accident and emergency department. Data on each patient were stored in a spreadsheet programme (Microsoft Excel, Microsoft Corporation, Redmond, Washington, USA) for later analysis. Confidence intervals (CI) for a proportion were calculated by the method described by Gardner and Altman. ${ }^{8}$

\section{Results}

During 1989, 773 patients were admitted with acute stroke which occurred in the previous seven days (excluding transient ischaemic attack, subarachnoid or subdural haemorrhage, and onset while hospitalised). There were 388 men and 385 women, whose mean (SD) age was $69.7(11 \cdot 7)$ years. Excluding 58 patients for whom the history was unclear or unavailable (mostly due to lack of a witness for a dysphasic or comatose patient), $63 \%$ (453/715) had arrived at the hospital within 12 hours, $76 \%(542 / 715)$ within 24 hours, and $85 \%(605 / 715)$ within 48 hours of the onset of symptoms.

CT of the brain was performed on 705 $(98.6 \%)$ of the 715 patients with adequate history. There were 364 patients with cerebral infarct, 137 with lacunar infarct, and 204 with intracerebral haemorrhage. Arrival time at the hospital varied significantly with stroke subtype (figure): at 12 hours, $44 \%$ (95\% CI $36 \%$ to $52 \%$ ) of patients with lacunar infarct, $61 \%$ (56\% to $66 \%$ ) with cerebral infarct, and $81 \%$ ( $76 \%$ to $86 \%$ ) with intracerebral haemorrhage had arrived at the hospital. At 24 hours, the corresponding proportions were $61 \%(52 \%$ to $69 \%), 73 \%$ (69\% to $78 \%$ ), and $90 \%(86 \%$ to $94 \%$ ); at 48 hours, they were $73 \%$ (66\% to $80 \%$ ), $83 \%$ ( $79 \%$ to $87 \%$ ), and $95 \%$ ( $92 \%$ to $98 \%)$ respectively.

\section{Discussion}

An important question in the planning of a stroke therapy trial is how many patients would present themselves early enough for treatment to begin within the designated time limit. There have been few studies, however, that quantify the delay in the presentation of patients with stroke. In a study of 457 patients 


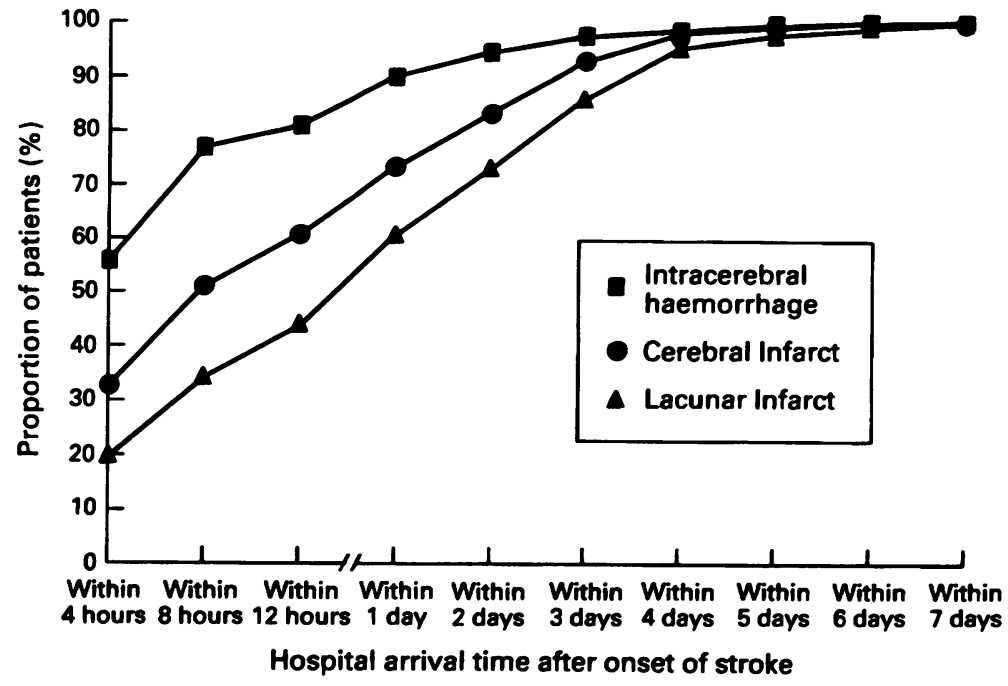

Figure Proportion of patients of each stroke subtype presenting at successive intervals after the onset of stroke. tions. ${ }^{5}$ This may partly explain why, on average, our stroke patients arrived at hospital earlier than their American counterparts. Other factors that might have encouraged our patients to attend hospital sooner could include: geographical compactness-no one in our catchment area lives more than $30 \mathrm{~km}$ from the hosipital; family support-old people usually live with the young who can take them to hospital; and health-seeking behaviourmany patients seek primary care direct from the hospital.

During this study, CT was performed routinely but with varying degrees of urgency. Only $51 \%$ of patients were scanned within 24 hours. When the therapeutic trial gets underway, CT will be arranged with more urgency. We therefore expect that about half of our patients with ischameic stroke may be included, as they would have presented to hospital within 12 hours of ictus.

1 Biller J, Love BB. Nihilism and stroke therapy (editorial). Stroke 1991;22:1105-7.

in North Carolina, USA, ${ }^{9}$ more than half of all patients with stroke did not present within 24 hours of onset, and one third did not present within 48 hours. In the United States Stroke Data Bank, ${ }^{10}$ conversely, about half of the patients were admitted by 12 hours and two thirds by 24 hours after onset. As for our Hong Kong patients, nearly two-thirds of them arrived at hospital within 12 hours of ictus, and three-quarters within 24 hours. Only $15 \%$ of our patients had not arrived within the first 48 hours.

The delay in presentation varied significantly within stroke subtype, the longest delay being observed in patients with lacunar infarct, and the shortest in those with intracerebral haemorrhage. In the Chinese population of Hong Kong, intracerebral haemorrhage accounts for approximately $30 \%$ of all strokes, in contrast to around $10 \%$ in Western popula- 